\title{
Idiopathic presenile cataract formation and galactosaemia
}

\author{
RHOADS E STEVENS,' MANUEL B DATILES, ${ }^{2}$ SATISH K SRIVASTAVA, ${ }^{3}$ \\ NASEEM H ANSARI, ${ }^{3}$ A EDWARD MAUMENEE, ${ }^{1}$ AND WALTER J STARK' \\ From the ${ }^{1}$ Wilmer Eye Institute of the Johns Hopkins Hospital, Baltimore, Maryland, and ${ }^{2}$ National Eye \\ Institute, National Institutes of Health, Bethesda, Maryland, and the ${ }^{3}$ University of Texas Medical Branch, \\ Galveston, Texas, USA
}

SUMMARY Five hundred patients undergoing cataract surgery were prospectively examined, and 46 Caucasian patients were found to have strictly idiopathic cataracts severe enough to warrant surgery on or before age 55. In a masked fashion we determined the activity of galactokinase (GK) and galactose-1-phosphate uridyl transferase (GPUT) in these patients as well as on 53 age matched controls. With respect to GK no cataract patient had an enzyme level of less than 2 standard deviations below the control mean. However, 3 of $45(6.7 \%)$ patients in the cataract group had a GPUT level less than 2 standard deviations below the mean for controls, and were presumably heterozygotes for this enzyme. In comparison with the expected population rate of $0.8 \%$ this is highly significant $(p=0.006)$. Abnormalities in galactose pathway enzymes may therefore predispose to development of presenile cataracts. In affected people there is a possibility of treating these patients clinically by dietary restriction of dairy products or by using aldose reductase inhibitors to prevent or reverse cataract formation.

Galactosaemia is an inborn error of metabolism characterised by inability to metabolise galactose due to absence of galactose-1- $\mathrm{PO}_{4}$ uridyl transferase (GPUT) or deficiency or absence of galactokinase (GK) (Fig. 1). Cataract formation is a primary manifestation in both enzyme defects.' However, classic galactosaemia is fatal unless galactose is withdrawn from the diet in early life.

Kinoshita ${ }^{2}$ and Cogan et al. ${ }^{3}$ have shown that cataract formation in galactosaemia is due to the accumulation of dulcitol through the action of the enzyme aldose reductase (AR) (Fig. 1, reaction 4). The polyol dulcitol does not readily pass through cell membranes and thus accumulates within lens fibres, including osmotic swelling. Subsequently there is severe derangement of metabolic activity, resulting in loss of amino acids, alteration of protein synthesis, changes in membrane permeability, and depression of adenosine triphospate (ATP) and reduced glutathione (GSH) levels. Finally fibres swell and rupture, resulting in cataract formation. Recent

Correspondence to Dr Manuel B Datiles, Clinical Branch, National Eye Institute-NIH, Building 10, Room 10N226, Bethesda, Maryland 20892, USA. studies also suggest that oxidative damage due to competition between aldose reductase and glutathione reductase for nicotinamide adenine dinucleotide phosphate (NADPH) may be another contributing factor in galactosaemic cataractogenesis.

Recently, potent aldose reductase inhibitors became available and were used as biological probes to determine the role of AR in galactosaemia and diabetes. It was found that galactose-induced cataracts in rats can be completely prevented by systemic treatment with sorbinil, a potent AR

1. Galactose + ATP $\stackrel{\text { Galactokinase }}{\longrightarrow}$ Galactose-1-phosphate + ADP

2. Galactose-1-phosphate + UDP glucose $\frac{\text { Galactose-1-phosphate }}{\text { uridyl transferase }}$

Glucose-1-phosphate + UDP galactose

3. UDP galactose $\stackrel{\text { UDP galactose-4-epimerase }}{=}$ UDP glucose

4. Galactose + NADPH $\stackrel{\text { Aldose reductase }}{=}$ Dulcitol + NADP

Fig. 1 Galactose metabolic pathway. 
inhibitor. ${ }^{4}$ Subsequently it was shown that systemic and topical application of sorbinil can reverse cataracts in galactosaemic animals. ${ }^{5}$ Interestingly new experiments have also shown that AR may be involved in other tissues, such as nervous, vascular (including retinal vessels), corneal, and renal tissues. $^{3}$

Heterozygotes for GK deficiency have approximately half the enzyme activity in the erythrocytes. ${ }^{\text {o- }}$ It was suggested that heterozygotes may be prone to early cataract formation. It has been estimated that GK heterozygosity occurs in $\mathbf{0 . 2} \%$ of the population and GPUT heterozygosity in $0.8 \%$ of the population. ${ }^{10}$ A study of patients with presenile cataracts found approximately half of the patients to have abnormalities in the galactose metabolic enzymes. ${ }^{911}$ Recent studies ${ }^{12}{ }^{13}$ have also shown a high prevalence of GK deficiency heterozygosity in patients with presenile idiopathic cataract.

Thus in many of the idiopathic presenile patients there may be an abnormality to metabolise galactose and hence predispose to cataract formation through the involvement of aldose reductase. The possibility exists of treating these patients clinically by restricting their dietary intake of dairy products or using aldose reductase inhibitors to prevent or reverse cataract formation.

We therefore undertook a case control study at the Wilmer Institute to determine the prevalence of abnormalities in the galactosaemic enzymes in patients with presenile cataract undergoing cataract surgery.

\section{Material and methods}

Five hundred consecutive patients scheduled for cataract surgery in the Wilmer Eye Institute Resident Service and those scheduled by two anterior segment surgeons (WJS and AEM) of the Wilmer Eye Institute of Johns Hopkins Hospital were carefully screened.

Inclusion criteria for this study were as follows: (1) Cataract surgery should have been performed or scheduled on or before age 55 . Since we used a strict definition of severe cataract requiring surgery by age 55 , we increased our age limit to 55, as Stambolian $e t$ al. ${ }^{13}$ did in their study. (2) Patients should come from within a radius of 100 miles from Baltimore. We wanted to limit the geographical area of the study in an effort to make our population geographically homogeneous.

Exclusion criteria were as follows: (1) Patients with a history of diabetes, treatment with corticosteroids, uveitis, ocular trauma, ocular radiation, myotonic dystrophy, lens subluxation, glaucoma, or congenital cataracts were excluded. (2) Black patients were excluded from the study. It cannot be assumed that reduced GK activity in a black person implies defect heterozygosity, since Tedesco and coworkers ${ }^{14}$ reported low GK levels in black patients in the absence of any cataract.

A control population was selected that matched the age, sex, race, and geographical characteristics of the cataract group. Patients with diabetes or myotonic dystrophy were excluded from the control group. Each control subject was screened for the absence of cataract. In general the control group consisted of patients from the Wilmer emergency room, friends and spouses of the cataract group, physicians, and hospital employees.

After obtaining verbal consent, venous blood was obtained from all cataract patients and controls by means of a sterile $7 \mathrm{ml}$ tube containing edetic acid (EDTA) (Vacutainer, lavender stopper, BectonDickinson, Rutherford, NJ). $0 \cdot 1 \mathrm{ml}$ of $50 \%$ dextrose in water was added to each sample immediately after collection in sterile fashion. The samples were labelled with the subject's name and date of collection and immediately stored at $4^{\circ} \mathrm{C}$. The samples were shipped on wet ice to the laboratory so as to arrive within five days of collection. Each shipment contained samples from cataract patients and control subjects. Laboratory staff performing the assays did not participate in specimen collection, nor did they know which specimens were from the cataract group and which were from the control group.

Erythrocytes were isolated by filtration through an alpha-cellulose and microcrystalline cellulose column. ${ }^{15}$ Assays for erythrocyte GK and GPUT activity were performed according to the methods of Beutler.$^{16}$ Because of laboratory error occasional assays were ruined, but they could not be repeated. These values were necessarily omitted from analysis. Data analysis was performed at the Wilmer Biostatistical Center.

\section{Results}

Five hundred cataract patients of appropriate age, race, and geographical location were screened to yield the study group. Most of these patients did not have 'idiopathic' cataracts. Of the patients that satisfied the study group criteria none refused to participate. However, seven could not be contacted, and four could not come in for venepuncture in accordance with the time constraints of the study. Forty-six patients with presenile idiopathic cataracts were entered into the study. Twenty-four (52\%) were male. The average age (SD) was $46(5.61)$ years with a range of 32 to 58 . The 58-year-old had undergone cataract surgery at age 55 for one eye. Twenty-four $(52 \%)$ of the patients were aged 50 or 
GALACTOKINASE

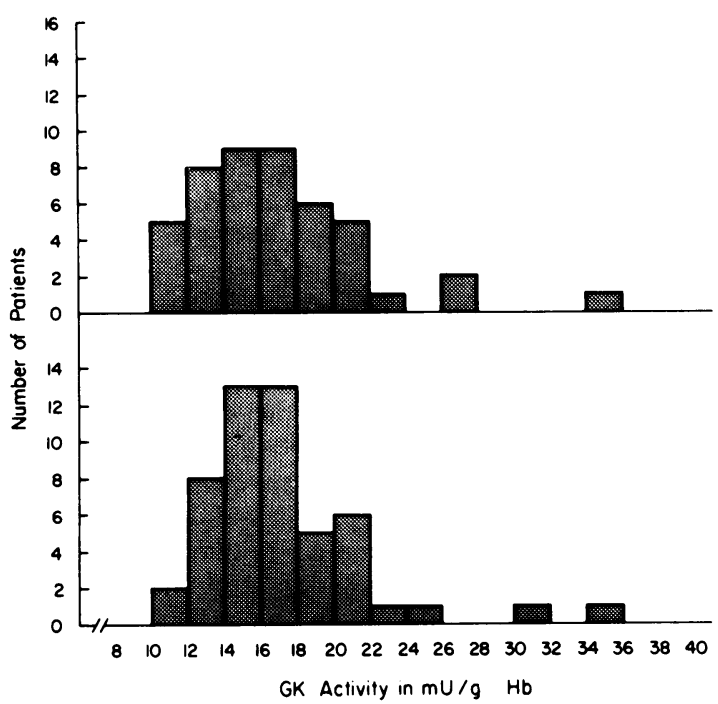

Fig. 2 Erythrocyte galactokinase activity for control and cataract groups. Cataract group: $n=46$, mean $=16 \cdot 80$, $S D=4 \cdot 61$. Control group: $n=51$, mean $=17 \cdot 55, S D=4 \cdot 42$.

younger at the time of venepuncture. Forty-four of the patients $(96 \%)$ had bilateral cataracts. Of the 90 cataracts $78(87 \%)$ had a posterior subcapsular opacity and $7(8 \%)$ did not. The type of cataract was unknown in $5(5 \%)$. Family history of cataract was very positive in 22 patients $(48 \%)$, equivocal in $4(9 \%)$, negative in $19(41 \%)$, and unknown in 1 $(2 \%)$.

The average duration of symptoms at the time of cataract surgery or examination was three years, with a range of three months to 11 years.

The control population consisted of 53 individuals, $31(58 \%)$ male and $22(42 \%)$ female. The average age of the control population was 44.4 (SD 6.6) years with a range of 30 to 55 .

While the average age of the control population was younger than that of the cataract group, a linear regression of GK and GPUT activity versus age for the control group produced slopes of only $\mathbf{0 . 0 6 1}$ and $-0.077(p=0.50)$ respectively. Since the control group enzyme activities do not significantly vary with age, this rather small difference in age between the control group and cataract group does not affect comparison between them.

Galactokinase activity determinations were performed on 46 cataract patients and 51 controls. These results are graphically depicted in Fig. 2 . The average activity for GK in the cataract group was $16.80(4.61)$ $\mathrm{mU} / \mathrm{g} \mathrm{Hb}$, while in the control group, the value was $17.35(4.42) \mathrm{mU} / \mathrm{g} \mathrm{Hb}$. There is no statistically

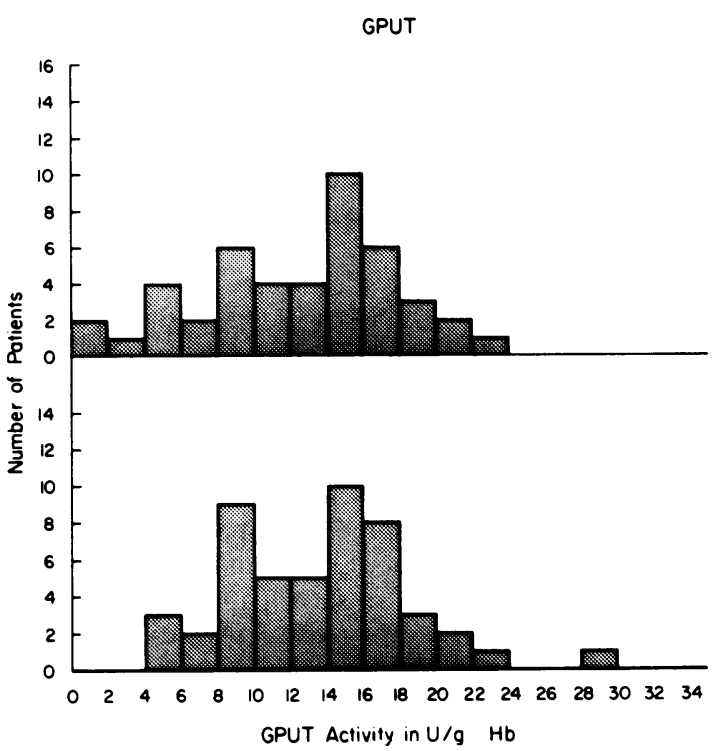

Fig. 3 Erythrocyte GPUT activity for control and cataract groups. Cataract group: $n=45$, mean $=12 \cdot 75, S D=5 \cdot 43$. Control group: $n=49$, mean $=13 \cdot 75, S D=4 \cdot 89$.

significant difference between these averages $(p=0 \cdot 60)$. No cataract patient had a GK activity that fell below 2 standard deviations from the average control value.

GPUT activity was determined on 45 cataract patients and 49 controls. These results are shown in Fig. 3. The average GPUT activity for the cataract group was $12.75(5.43) \mathrm{U} / \mathrm{g} \mathrm{Hb}$. This did not statistically differ from the control average of $13.75(4.89)$ $\mathrm{U} / \mathrm{g} \mathrm{Hb}(\mathrm{p}=0.93)$. However, three of the 45 cararact patients $(6.66 \%)$ had a GPUT activity that fell below 2 standard deviations from the control group average, which is a significantly high percentage of abnormals, given the population rate of $0 \cdot 8 \%$ $(\mathrm{p}=0.006$, Poisson probability).

\section{Discussion}

True idiopathic presenile cataracts are relatively rare, and this parameter must be carefully monitored by an investigator attempting to study this population. Although our screening population included children, none had idiopathic cataracts. The cataracts were either congenital, traumatic, or associated with other ocular diseases. Children, if they do have galactosaemia, are more apt to be homozygous, not heterozygous. Thus one would not expect them to appear in the cataract group in this study.

The comparison of concurrent controls was also 
important in this particular study, since the estimated gene frequency of either GK or GPUT enzyme defect in the general population is extremely low. One would not expect the mean value of either enzyme to be different from the controls' unless heterozygotes made up a large part of the cataract group. However, the fact that the percentage of heterozygotes was insufficient to make the mean enzyme levels significantly lower than those of the controls in either group does not exclude the role of GPUT levels in the aetiology of idiopathic cataracts.

In this study no cataract patient had GK levels less than 2 standard deviations below the mean for controls. These results are contrary to the findings of Skalka and Prchal ${ }^{911}$ and Stambolian et al. ${ }^{13}$ who reported, respectively, $37 \%$ and $7-8 \%$ patients with galactokinase activity more than 2 standard deviations below the avarage. This negative result, given the $0.2 \%$ frequency for the heterozygote, is not unexpected and may easily reflect small sample size. However, in agreement with earlier investigators there were $6.7 \%$ patients (three out of 45 ) in the cataract group with significantly low GPUT levels. Although we did not find GK deficiency in our presenile cataract patients, the deficiency of GPUT strongly suggests a role of galactose pathway enzymes in the aetiology of idiopathic cataracts and supports previous work. ${ }^{91213}$

Hence in dealing with patients with idiopathic presenile cataracts it may be worth exploring the role played by the galactose pathway enzymes. Once affected individuals are identified, they and their families may benefit from restriction of dairy products in the diet and possibly from treatment with aldose reductase inhibitors.

This study was supported in part by grant EY-01677 (SK Srivastava).

\section{References}

1 Isselbacker KJ. Galactosemia. In: Wyngaarden JB, Fredrickson DS, eds. The metabolic basis of inherited disease. 2 nd ed. New York: McGraw-Hill, 1966: 178-88.

2 Kinoshita JH. Mechanisms initiating cataract formation. Invest Ophthalmol Vis Sci 1974; 13: 713-24.

3 Cogan D, Kinoshita JH, Kador P, et al. Aldose reductase and complications of diabetes. Ann Intern Med 1984; 101: 82-91.

4 Datiles M, Fukui H, Kuwabara T, Kinoshita JH. Galactose cataract prevention with sorbinil, an aldose reductase inhibitor: a light microscopic study. Invest Ophthalmol Vis Sci 1982; 22: 174-9.

5 Hu TS, Datiles M, Kinoshita JH. Reversal of galactose cataract with sorbinil in rats. Invest Ophthalmol Vis Sci 1983; 124: 640-4.

6 Beutler E, Krill A, Comings D, et al. Galactokinase deficiency: an important cause of familial cataracts in children and young adults. J Lab Clin Med 1970; 76: 1006.

7 Levy NS, Krill AE, Beutler E. Galactokinase deficiency and cataracts. Am J Ophthalmol 1972; 74: 41-8.

8 Monteleone JA, Beutler E, Monteleone PL, et al. Cataracts, galactosemia, and hypergalactosemia due to galactokinase deficiency in a child: studies of a kindred. Am J Med 1971; 50: 403-7.

9 Skalka HW, Prchal JT. Presenile cataract formation and decreased activity of galactosemia enzymes. Arch Ophthalmol 1980; 98: 269-73.

10 Levy HL, Hammersen G. Newborn screening for galactosemia and other galactose metabolic defects. J Pediatr 1978; 92: 871-7.

11 Skalka HW, Prchal JT, Conrad ME. Cataract formation in patients with decreased activity of galactosemic enzymes. Metab Pediatr Syst Ophthalmol 1979; 3: 97-101.

12 Elman MJ, Miller MT, Matador R. Galactokinase activity in patients with idiopathic cataracts. Ophthalmology 1986; 93: 210-5.

13 Stambolian D, Scarpino-Meyers V, Eagle RC, Hodes B, Harris H. Cataracts in patients heterozygous for galactose deficiency. Invest Ophthalmol Vis Sci 1986; 27: 429-33.

14 Tedesco TA, Bonow R, Miller K, Mellman N. Galactokinase: evidence of a new racial pholymorphism. Science 1972; 178: 176-8.

15 Beutler E, West C, Blume KG. The removal of leucocytes and platelets from whole blood. J Lab Clin Med 1976; 88: 328-33.

16 Beutler E. Red cell metabolism. New York: Grune and Stratton, 1971: 79-88.

Accepted for publication 19 November 1987. 